\title{
An X-Band Low Noise Amplifier Design for Marine Navigation Radars
}

\author{
Christina Lessi1, Evangelia Karagianni ${ }^{1,2}$ \\ ${ }^{1}$ National and Kapodistian University of Athens, Athens, Greece \\ ${ }^{2}$ Hellenic Naval Academy, Pireaus, Greece \\ Email: chrislessiee@gmail.com, evka@hna.gr
}

Received 3 February 2014; revised 1 March 2014; accepted 8 March 2014

Copyright (C) 2014 by authors and Scientific Research Publishing Inc.

This work is licensed under the Creative Commons Attribution International License (CC BY). http://creativecommons.org/licenses/by/4.0/

(c) (i) Open Access

\begin{abstract}
In this paper, the design of a $9.1 \mathrm{GHz}$ Low Noise Amplifier (LNA) of a RADAR receiver that is used in the Navy is presented. For the design of the LNA, we used GaAs Field-Effect Transistors (FETs) from Agilent ADS component library. In order to keep the cost of the circuit in low prices and the performance high, we design a two-stage LNA.
\end{abstract}

\section{Keywords}

Navy RADAR; Low Noise Amplifier (LNA); Field Effect Transistor (FET)

\section{Introduction}

RADARs are the eyes of observers in circumstances where the visibility is poor or nonexistent. Their use is necessary in sectors such as marine or air navigation. The most common type of a Marine Navigational Radar is the Monostatic Radar. Even though the specifications of such an implementation differentiate regarding its use, the high transmitter emitting power, the high gain of its antenna and the high sensitivity of the receiver comprise some of the standard requirements that the marine navigation radar should have. From Equation (1), it is evident that the satisfaction of the aforementioned radar characteristics leads to a longer operation range. More specifically, $P_{t}$ and $P_{r}$ are the transmitted and received power, $G$ is the antenna gain, $A_{e}$ is the effective aperture (area) of the antenna, $\sigma$ is the radar cross section of the target and $R$ is the distance where a target can be detected.

$$
P_{r}=\frac{P_{t} \cdot G \cdot A_{e} \cdot \sigma}{(4 \pi)^{2} \cdot R^{4}} \text { or } R_{\max }=\sqrt[4]{\frac{P_{t} \cdot G \cdot A_{e} \cdot \sigma}{(4 \pi)^{2} \cdot P_{r, \text { min }}}}
$$

The LNA is one of the most important building blocks of the receiver, since it is responsible in properly amplifying the received data for the following detection process. For this reason the intrinsic Noise Figure (NF) of 
such an amplifier plays the most important role for the total system NF in $\mathrm{dBs}$ ( $F_{\text {system }}$ pure number), as shown in (2), where $F_{i}$ and $G_{i}$ are the NF and the gain respectively of each device of the system [1]-[5]. So the first limitation for our design (our target) is the achievement of $7 \mathrm{~dB}$ NF. Of course it is important for the amplifier to amplify the input signal. So the gain should be at least $15 \mathrm{~dB}$ (second limitation). In addition, it should be kept in low values of the input port voltage reflection coefficient $(|\mathrm{S} 11|<-6 \mathrm{~dB})$ and the output port voltage reflection coefficient $(|\mathrm{S} 22|<-6 \mathrm{~dB})$. Voltage standing wave ratio (VSWR) both at input and output should be lower than $3 \mathrm{~dB}$.

$$
F_{\text {system }}=F_{1}+\frac{F_{2}-1}{G_{1}}+\cdots+\frac{F_{n}-1}{G_{1} \cdot G_{2} \cdots \cdot G_{n-1}}
$$

From the first of July 2002 where it is mandatory to fit two radars, one must be $\mathrm{X}$ Band (9 GHz) and one must be $\mathrm{S}$ Band $(3 \mathrm{GHz})$ unless the local administration states that two $\mathrm{X}$ band radars are permissible. All new buildings must comply with the new carriage rules. The $\mathrm{X}$ band radar, transmitting in higher performance, has better sensitivity and tracking performance; it can better discriminate two small targets close to each other, and it can also portray the coastlines better. It also gives a higher gain for same antenna dimensions. On the other hand higher frequencies are attenuated more in atmosphere, particularly in adverse conditions of fog, rain and sea clutter and are amplified with more difficulty [6].

For the design of the LNA we have chosen the GaAs FETs since it operates better than the Si BJT in high radio frequencies. Despite the fact that SiGe Heterojunction Bipolar Transistors (HBT) have been reported in the literature as a cheaper solution with proper performance in the high frequencies region similar to GaAs FETs [1]-[3], we have chosen not to use this type of transistor due to its lack of manufacturing maturity. In order to address the low cost constrains of the LNA chip, we focused on limiting the amplification stages in two [5]. Our effort is focused on achieving high enough gain and low noise figure [7] [8].

\section{Design}

The first step in our LNA design was the confirmation that the transistor is unilateral, and that satisfies (3).

$$
\frac{1}{(1+U)^{2}}<\frac{G_{T}}{G_{T U}}<\frac{1}{(1-U)^{2}}
$$

where $G_{T}$ and $G_{T U}$ are the transducer and the unilateral transducer gain $\left(S_{12}=0\right)$ respectively and

$$
U=\frac{\left|S_{11}\right| \cdot\left|S_{12}\right| \cdot\left|S_{21}\right| \cdot\left|S_{22}\right|}{\left(1-\left|S_{11}\right|^{2}\right)\left(1-\left|S_{22}\right|^{2}\right)}
$$

For low noise applications the transistors are usually biased at low current levels, since in that way the minimum possible noise is achieved; however with the cost of a limited amplification gain. In order to achieve higher amplification ability, thus amplifier gain, we have decided to implement an additional amplification level at our LNA design. The transistors' topology that has been selected was the cascaded one as presented in Figure 1.

For the purpose of ensuring a lower noise, the matching circuits designed. For the input matching circuit we used the Smith chart to define the starting point as close to the $\Gamma_{\mathrm{OPT}}$ on the Smith chart (Figure 2).

For the design of the output matching circuit, the input matching circuit was inserted in the circuit of the amplifier and the goal has been $\Gamma_{\text {Out }}$ to reach the center of the Smith chart (Figure 3). Finally the matching circuit which is intermediate to the two LNA stages should connect $Z_{\text {OUT1 }}$ to $Z_{\text {OUT2 }}$ [9] (Figure 4 ) ( $\Gamma$ is the reflection coefficient and $\mathrm{Z}$ is the impedance).

Finally, the resulting circuits were added in the total LNA circuit. After several simulations we have decided to make some changes in the values of the capacitances and the henries of the matching circuits of our final LNA circuit. These changes derived from the parasitic capacitances and inductances that could not be pre-estimated [10] [11]. Figure 1 shows the final LNA circuit. Apart from the three matching circuits (input, output and intermediate), there are two $100 \mathrm{pF}$ capacitors which are the DC-Blocks.

\section{Results and Discussion}

The simulation results have shown a $20.1 \mathrm{~dB}$ gain that is higher than the goal gain of the $15 \mathrm{~dB}$, as depicted in 


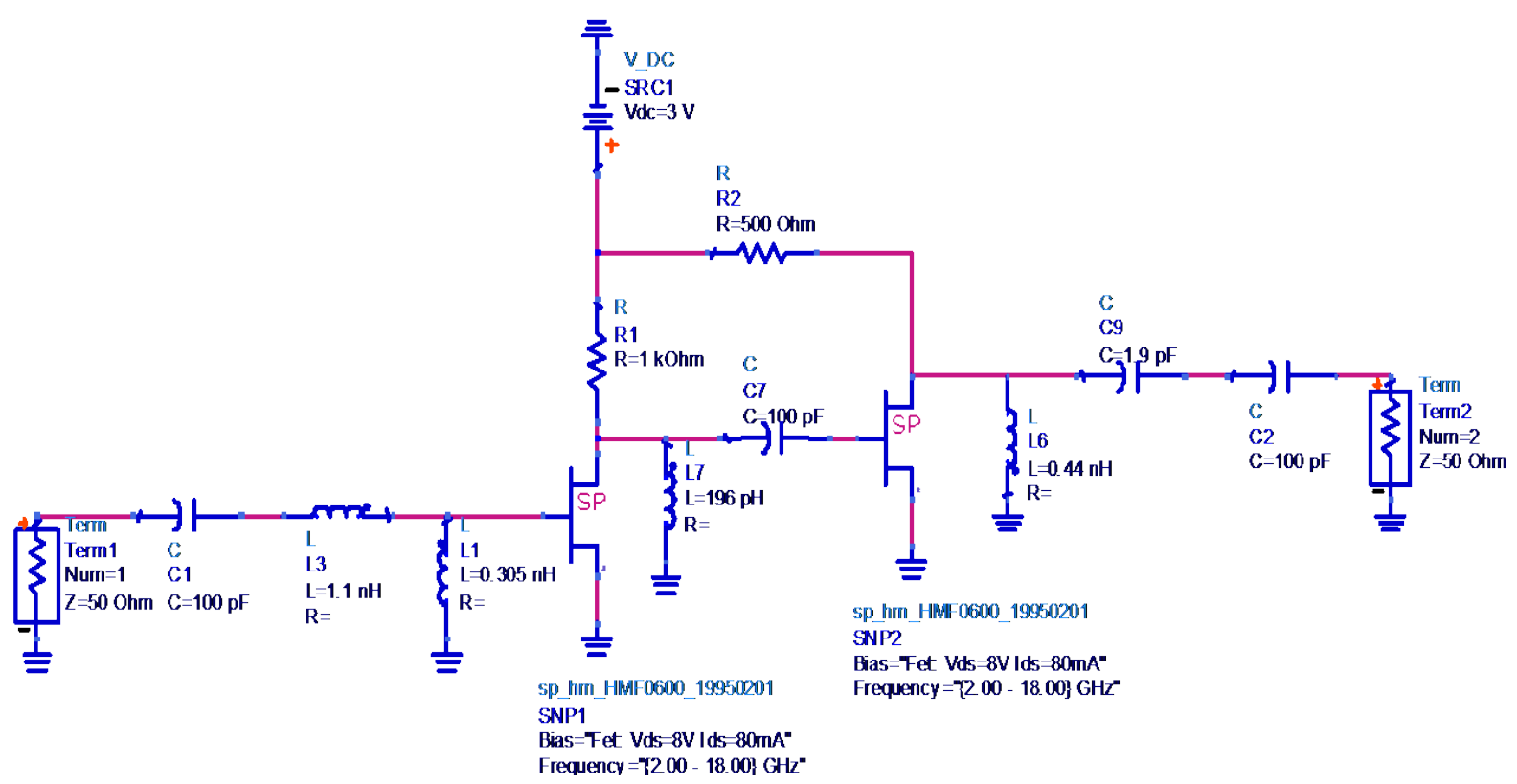

Figure 1. Final LNA circuit.

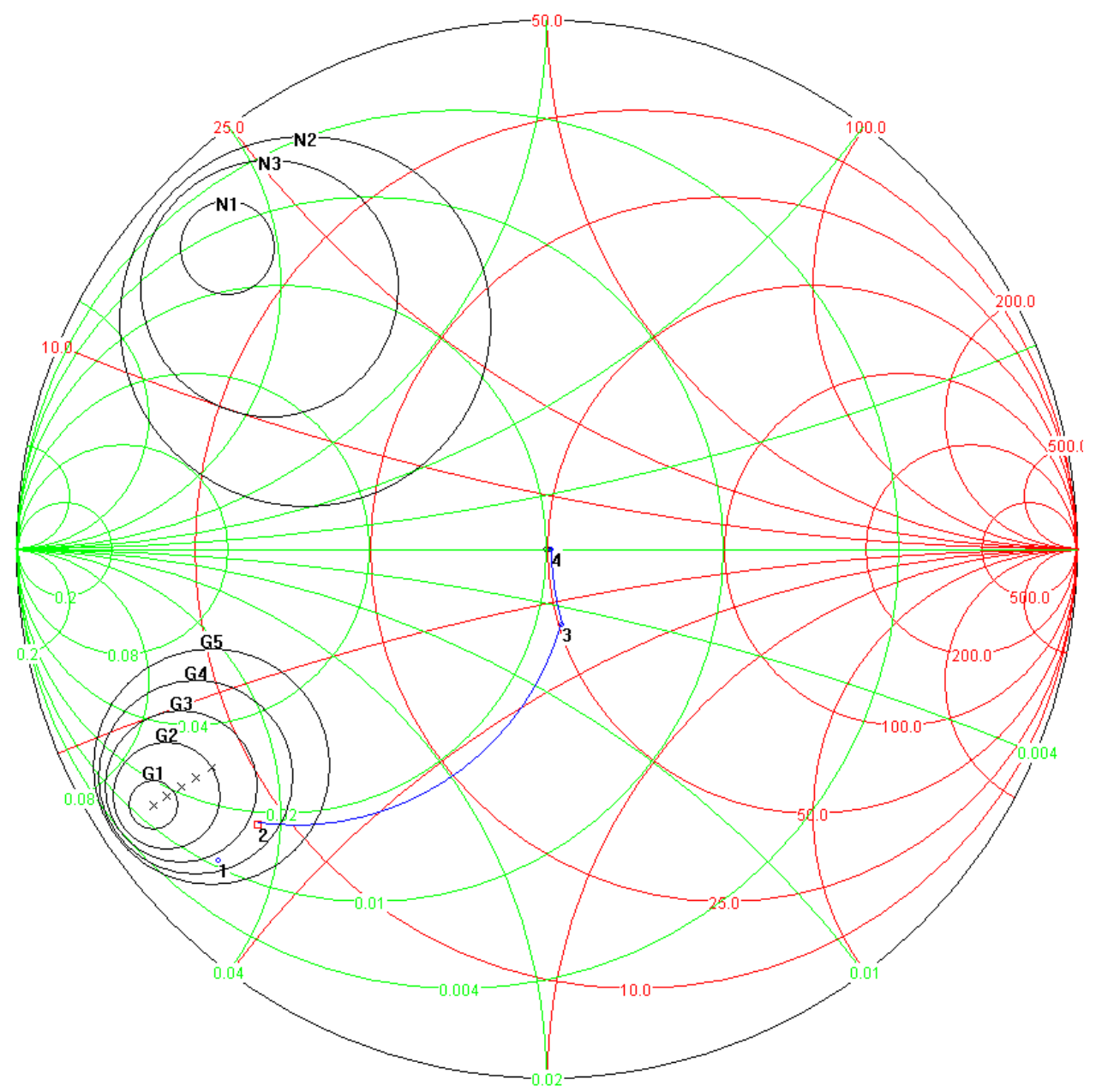

Figure 2. Input matching circuit. 


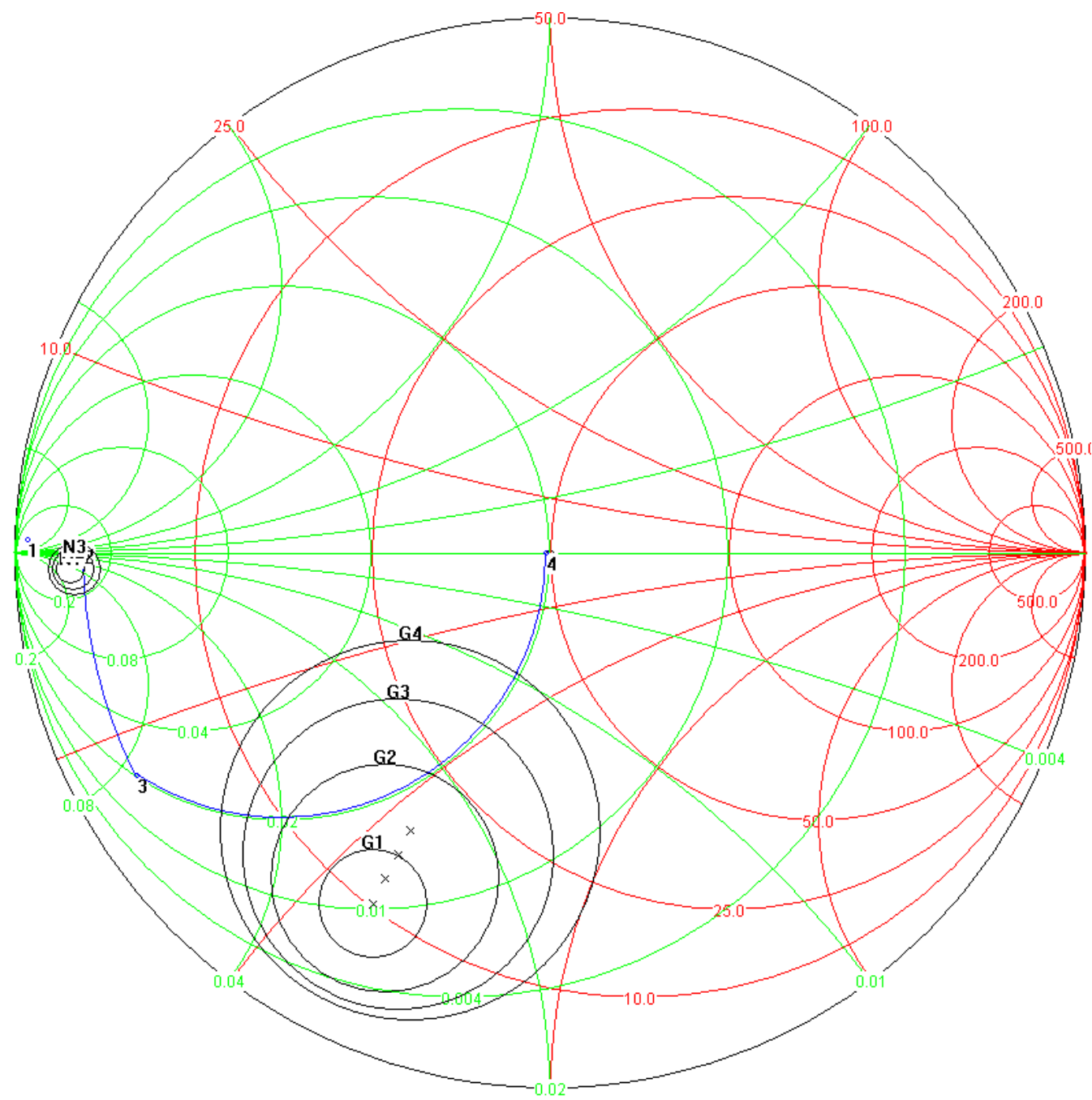

Figure 3. Output matching circuit.

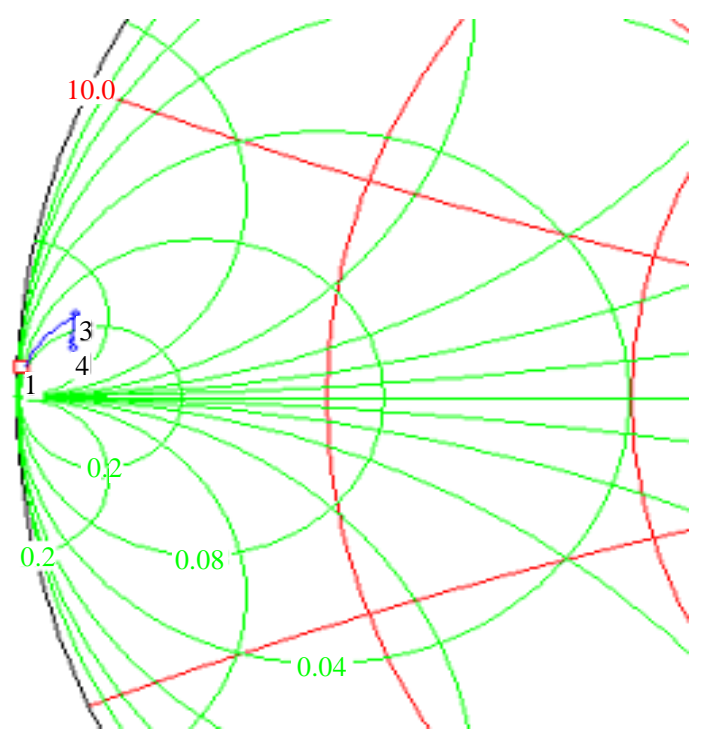

Figure 4. Intermediate matching circuit. 
Figure 5. More specifically, as it is noticed a slight frequency shifting of the received signal will not affect the gain significantly.

Moreover, in Figure 6 the S11 and S22 are presented. Their values are $-9.795 \mathrm{~dB}$ and $-15.121 \mathrm{~dB}$ respectively at the region of the $9.1 \mathrm{GHz}$. The lower value of S22 was found to be $-30.534 \mathrm{~dB}$ at $9.3 \mathrm{GHz}$, however this behavior is acceptable due to the LNA high performance at the $9.1 \mathrm{GHz}$ region. Additionally it should be noted that the LNA response at the $9.3 \mathrm{GHz}$ could be helpful especially in the case where a slight frequency shifting of the received signal would occur.

Figure 7 shows the NF response of the two-stage LNA and as noticed it reaches $\sim 6.9 \mathrm{~dB}$, which is lower than the $7 \mathrm{~dB}$ acceptance NF limit. Also, both input and output VSWR were measured to be lower than $3 \mathrm{~dB}$, with the input and the output VSWR to be 1.958 (Figure 8) and $1.425 \mathrm{~dB}$ (Figure 9) respectively.

It should be noted that an amplifier should be stable not only at its operational frequency, but also in a wide range of input frequencies. This stems from the fact that the output load of the LNA may be different compared to the simulation. Figure 10 presents the input stability circles and Figure 11 the output stability circles for frequencies between $4 \mathrm{GHz}$ and $14 \mathrm{GHz}$ with a step of $0.1 \mathrm{GHz}$. As shown, the LNA is stable in all these frequencies since the stability circles are both out of the unitary Smith chart and simultaneously the center of Smith chart is stable (both the $\mid \Gamma$ in $\mid$ and the $\mid \Gamma$ out $\mid$ are lower than 1).

Even though the performance of the simulated LNA is efficient, the accretion of additional amplification stages would improve the overall LNA performance. However it should be noted that the addition of an extra component increases the total circuit cost and its energy consumption.

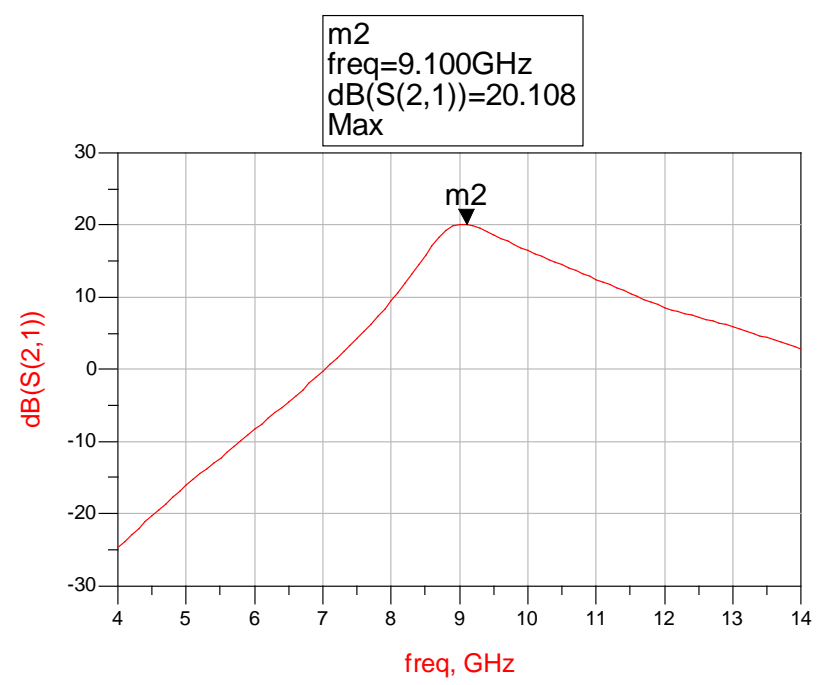

Figure 5. The gain of the LNA.

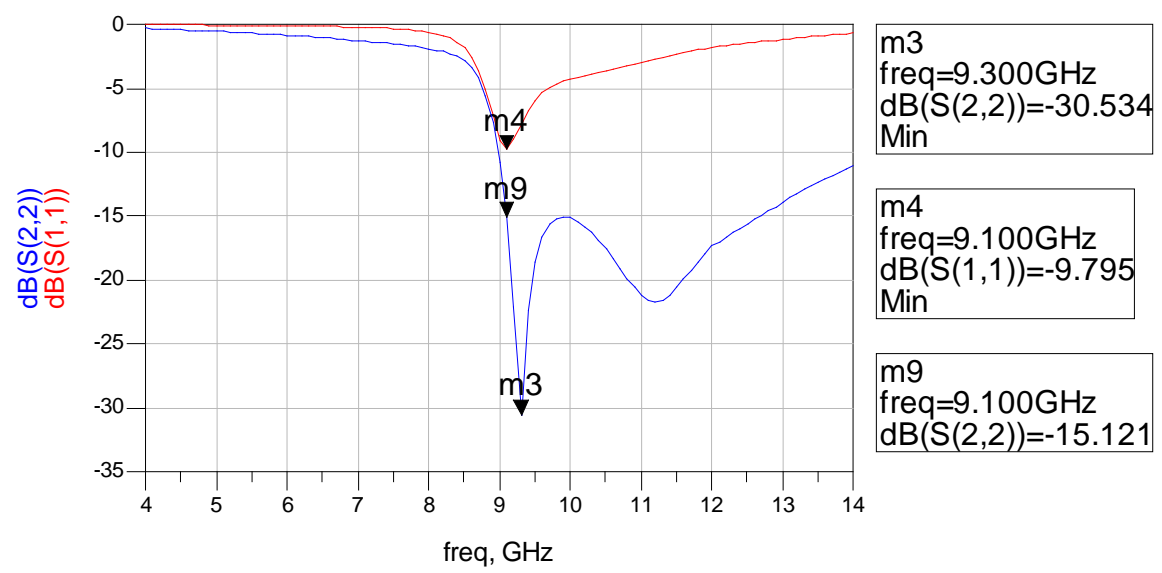

Figure 6. S11 and S22 LNA parameters. 


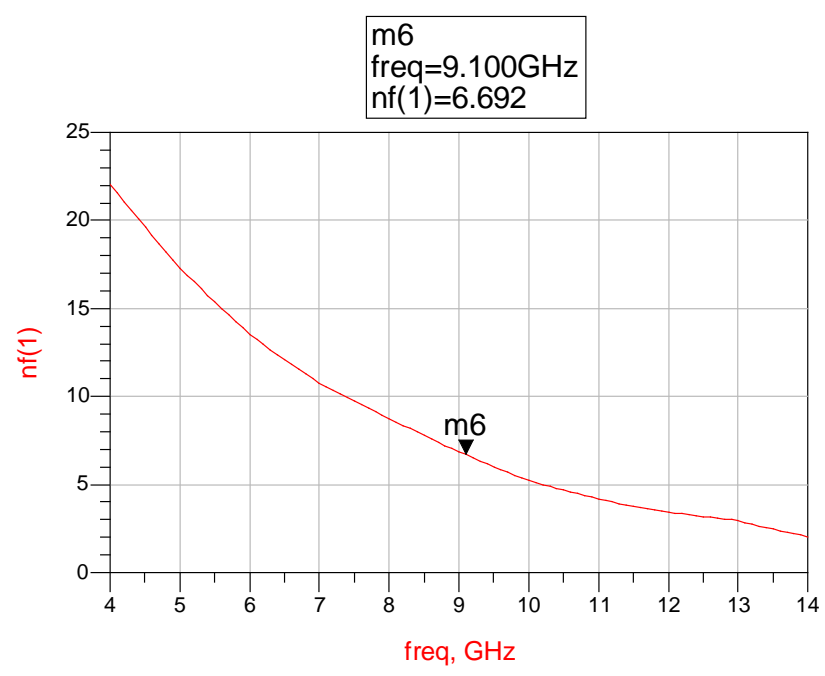

Figure 7. LNA Noise Figure response.

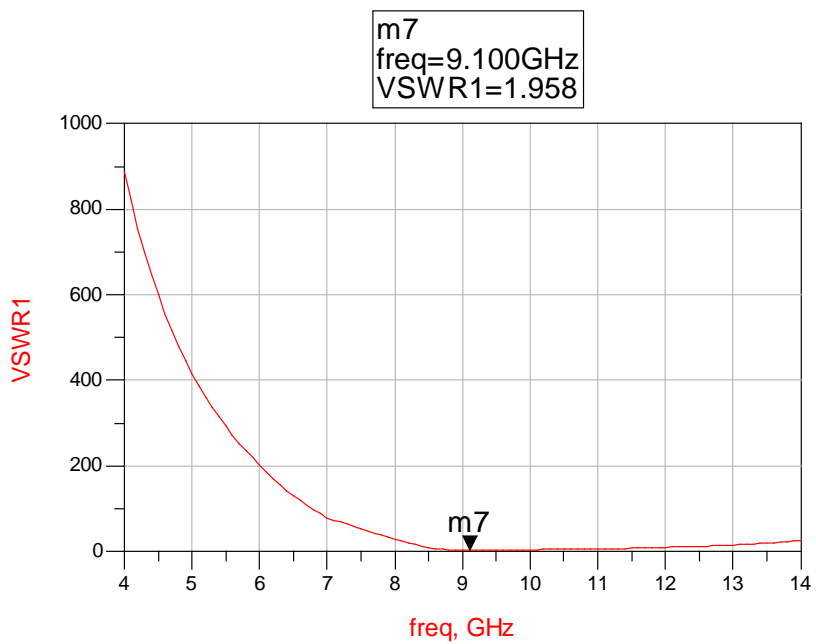

Figure 8. LNA input VSWR.

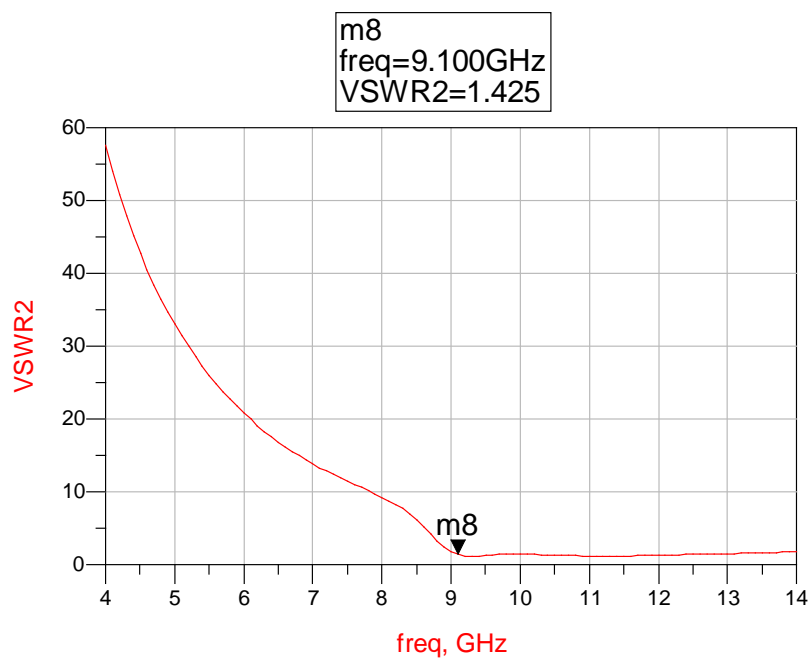

Figure 9. Output VSWR of the LNA. 


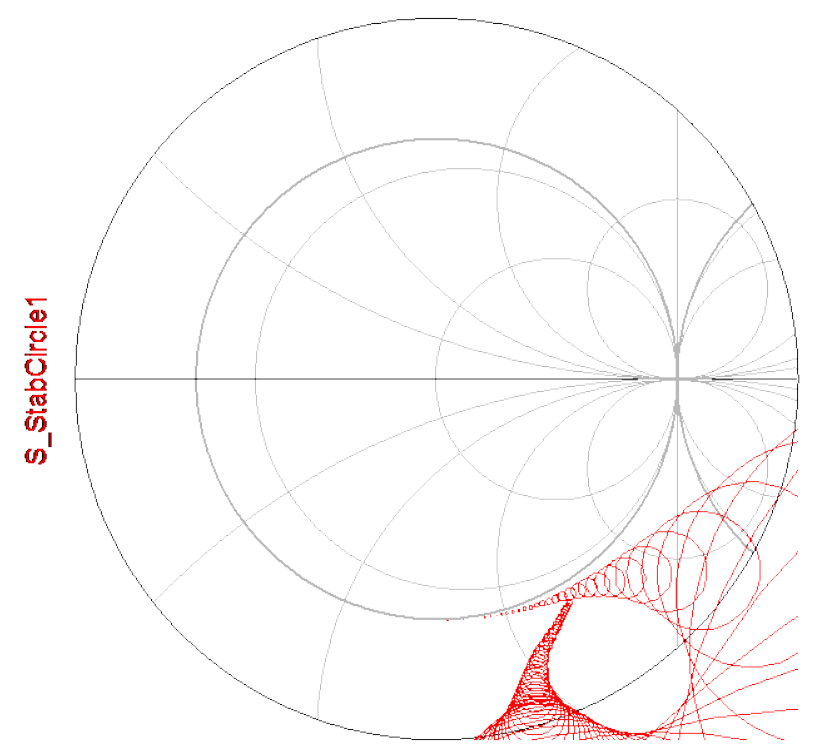

Figure 10. LNA input stability circles at $4 \mathrm{GHz}$ to $14 \mathrm{GHz}$ with $0.1 \mathrm{GHz}$ step.

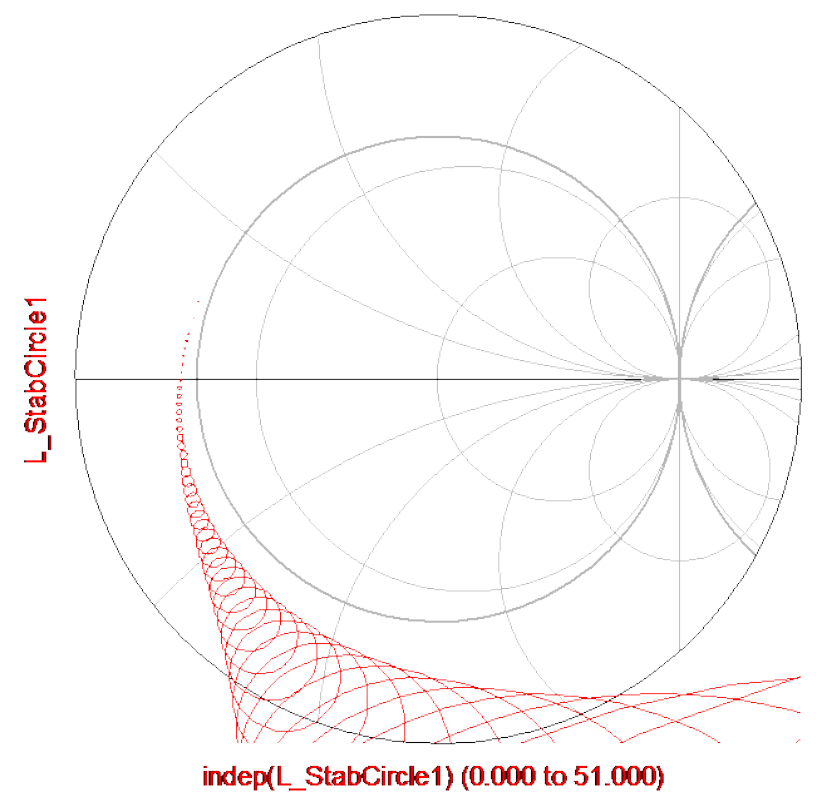

Figure 11. LNA output stability circles at $4 \mathrm{GHz}$ to $14 \mathrm{GHz}$ with $0.1 \mathrm{GHz}$ step.

\section{Conclusion}

We have designed a two-stage cascade GaAs LNA operating at $9.1 \mathrm{GHz}$ which is attractive for Navy Radar applications. We have showed that the LNA is stable in a wide span of frequencies between $4-14 \mathrm{GHz}$. Its gain and NF were measured to be 20.108 and $6.92 \mathrm{~dB}$ respectively. In addition, both input and output VSWR were lower than $3 \mathrm{~dB}$. Finally, S11 parameter was $-9.975 \mathrm{~dB}$ and the S22 was $-15.121 \mathrm{~dB}$.

\section{Acknowledgments}

The authors acknowledge that this work was partially supported by the State Scholarships Foundation through the European Social Fund and the National Strategic Reference Framework (2007-2013). 


\section{References}

[1] Kuo, W.-M.L., et al. (2006) An X-Band SiGe LNA with 1.36 dB Mean Noise Figure for Monolithic Phased Array Transmit/Receive Radar Modules. IEEE Radio Frequency Integrated Circuits (RFIC) Symposium, San Francisco, 11-13 June 2006, 501.

[2] Masuda, T., et al. (2005) SiGe HBT Based 24-GHz LNA and VCO for Short-Range Ultra-Wideband Radar Systems. Asian Solid-State Circuits Conference, Hsinchu, 1-3 November 2005, 425-428.

[3] Dinc, T., et al. (2013) Building Blocks for an X-Band SiGe BiCMOS T/R Module. IEEE Topical Conference on Power Amplifiers for Wireless and Radio Applications (PAWR), Austin, 20-23 January 2013, 97-99.

[4] Colangeli, S., et al. (2013) GaN-Based Robust Low-Noise Amplifiers. IEEE Transactions on Electron Devices, 60, 3238-3248. http://dx.doi.org/10.1109/TED.2013.2265718

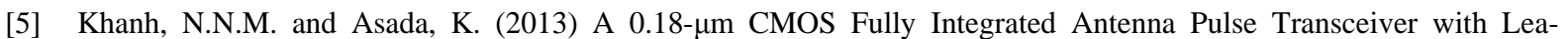
kage-Cancellation Technique for Wide-Band Microwave Range Sensing Radar. IEEE Radio Frequency Integrated Circuits Symposium (RFIC), Seattle, 2-4 June 2013, 97-100.

[6] Karagianni, E. and Gyparis, I. (2013) Telecommunication and Navigation Systems: A Monography for ETO Training according to STCW.

[7] Homaee, A. (2013) A CMOS 3.1 - 10.6 GHz UWB LNA Employing Modified Derivative Superposition Method. Circuits and Systems, 4, 323-327. http://dx.doi.org/10.4236/cs.2013.43044

[8] Kapsides, J.D., Chryssomallis, M.T. and Kyriacou, G.A. (2000) A Technique for the Design of Microwave Transistor Oscillators for Optimum Power Output. Proceedings of the Second International Symposium of Trans Black Sea Region on Applied Electromagnetism, Xanthi, 27-29 June 2000. http://dx.doi.org/10.1109/AEM.2000.943257

[9] Pozar, D.M. (1998) Microwave Engineering. John Wiley \& Sons, Hoboken.

[10] Hsu, M.-T., Du, J.-H. and Chiu, W.-C. (2012) Design of Low Power CMOS LNA with Current-Reused and Notch Filter Topology for DS-UWB Application. Wireless Engineering and Technology, 3, 167-174. http://dx.doi.org/10.4236/wet.2012.33024

[11] Wu, C.-S., Lin, T.-Y., Chang, C.-H. and Wu, H.-M. (2011) 3 - 10 GHz Ultra-Wideband Low-Noise Amplifier Using Inductive-Series Peaking Technique with Cascode Common-Source Circuit. Wireless Engineering and Technology, 2, 257-261. http://dx.doi.org/10.4236/wet.2011.24034 